\title{
Análisis del fenómeno de niños, niñas y adolescentes en situación de trabajo en la ciudad de Villavicencio*
}

\section{Analysis of the phenomenon of children and adolescents in work situation in the city of Villavicencio}

\author{
Gabriel Balaguera Rojas \\ ORCID: 0000-0003-1121-8972
}

Mónica del Pilar Balaguera Rojas*

ORCID: 0000-0003-3786-5553

Universidad Santo Tomás, Colombia

Recibido: 17 de marzo de 2018

Revisado: 6 de abril de 2018

Aceptado: 4 de mayo de 2018

\section{Resumen}

El fenómeno de los niños, niñas y adolescentes en situación de trabajo en Colombia es foco de atención de instituciones gubernamentales y no gubernamentales por lo que es importante generar estrategias que mitiguen su proliferación ya que se afectan derechos fundamentales. Esta situación es objeto de investigación en la ciudad de Villavicencio en el Departamento del Meta; por lo que a través de las verbalizaciones de dos grupos de interés que para el caso fueron los niños, niñas y adolescentes $(n=24)$ que se encontraban desarrollando actividades de índole laboral por un lado, y por otro, los integrantes del Comité de Erradicación del Trabajo Infantil CETI de dicha ciudad $(n=24)$. Como resultado de la investigación se observa que hay similitudes y diferencias en las verbalizaciones respecto al fenómeno y como se presenta, ya que se observan discrepancias significativas entre lo que las instituciones dedicadas a erradicar el fenómeno y las actividades que realizan los menores.

Palabras clave: trabajo infantil, menores en situación de trabajo, instituciones de erradicación de trabajo infantil.

Artículo de investigación. Citar como: Balaguera, R. G. \& Balaguera, R. M. (2019) Análisis del fenómeno de niños, niñas y adolescentes en situación de trabajo en la ciudad de Villavicencio. Diversitas: Perspectivas en Psicología, 15(1), 11-25. DOI: https://doi.org/10.15332/s1794-9998.2019.0001.01 Correspondencia: Mónica Balaguera Rojas, Docente Facultad de Psicología. Universidad Santo Tomás. Grupo de Investigación Psicología, ciclo vital y derechos. Dirección postal: Campus San Alberto Magno, Autopista Norte calle 205, vía Arrayanes km 1.6. Bogotá, Colombia Correo electrónico: monicabalaguera@usantotomas.edu.co 


\section{Abtract}

The phenomenon of children and adolescents in work situations in Colombia is a focus of attention of governmental and no governmental institutions, so this is an important issue to generate strategies that mitigate its proliferation since Fundamental Rights are affected, this situation is the subject of research in the city of Villavicencio in the Department of Meta; so through the verbalizations of two interest groups that for the case were the children and adolescents who were actually developing activities of a labor nature and on the other hand, the members of the Committee for the Eradication of Child Labor CETI; therefore, as results of the investigation, it is observed that there are similarities and differences in the verbalizations regarding the phenomenon and how it is presented.

Keywords: child labor, minors in work situation, child labor eradication institutions.

\section{Introducción}

Las dinámicas sociales, familiares, culturales e históricas producto de las relaciones interpersonales y de la cotidianidad humana, exigen en el diario vivir de muchos niños, niñas y adolescentes (en adelante NNA) participar en actividades laborales denominadas trabajo infanto-juvenil. Sin embargo, dada la constante vulneración de algunos de sus derechos fundamentales según lo mencionan Avella y Medina (2006), repercuten en la salud física y mental y además genera mayor pobreza a la sociedad (Nova, 2008).

Lo anterior, cobra relevancia dado que los NNA que se encuentran en situación de trabajo realizan labores que no son propias de su etapa de desarrollo, lo que conlleva a efectos desfavorables y con esto no se cumple lo suscrito en la Constitución Política de Colombia respecto al tema (Avella \& Medina, 2006).

El interés de la presente investigación se fundamenta en atender los derechos de los NNA y su vulneración; que en muchas ocasiones es violentada a partir del desconocimiento o desatención de la normatividad vigente, que vela por la seguridad de la población en cuestión. Esta regulación busca evitar que los NNA sean víctimas de explotación o de incorrectas rutas de atención por parte de los entes reguladores encargados, lo cual se debe a la falta de claridad en el proceso que se debe realizar ante ese tipo situaciones (Asamblea Constituyente, 1991).

Por otra parte es importante destacar que los NNA deben contar con las condiciones mínimas en cuanto al bienestar integral y que en la actualidad no existe consenso acerca de la forma adecuada para abordar el problema del trabajo infantil y juvenil en Colombia. Así pues, sin importar cuál sea la causa por la cual el menor se encuentre laborando, ésta actividad laboral es generadora de consecuencias negativas para el menor, por lo que se debe propender por plantear alternativas con el fin de brindarle las condiciones de bienestar que merece.

Es así, que la problemática pretende ser abordada desde la perspectiva del trabajo informal, a partir de la información de la normatividad colombiana. Esta normatividad regula la actividad objeto de estudio de la presente investigación y las instituciones que la hacen cumplir. Por lo tanto, surge la siguiente pregunta de investigación ¿son concordantes las estrategias que tiene el Estado colombiano, a través de sus instituciones respecto la realidad del fenómeno de niños, niñas y adolescentes en situación de trabajo en la ciudad de Villavicencio-Meta?

Por lo mencionado anteriormente, se plantea como objetivo general, identificar las realidades a cerca del fenómeno de los NNA en situación de trabajo y las concepciones que tienen las instituciones 
encargadas de erradicar el trabajo infanto-juvenil en la ciudad de Villavicencio (Meta) a través de las verbalizaciones de los actores involucrados en el fenómeno como lo son los NNA en situación de trabajo y las instituciones estatales responsables de su control. Para llevar a cabo esto se plantean como objetivos específicos los siguientes. En primer lugar, categorizar las verbalizaciones emergentes de los dos grupos poblacionales. Luego, contrastar las realidades emergentes enunciadas por cada grupo actor respecto a las actividades y finalmente establecer las diferencias y similitudes a partir de las realidades verbalizadas y los objetivos que persiguen las instituciones responsables ante situaciones de trabajo infantil.

La presente investigación entiende al trabajo infantil como cualquier actividad que involucre la comercialización, producción, transformación, distribución o venta de bienes o servicios; teniendo en cuenta remuneración o no, o se realice en forma independiente $\mathrm{o}$ al servicio de otra persona natural o jurídica por parte de personas que no han cumplido los 18 años de edad. Desafortunadamente esta es una de las realidades que afecta principalmente a los países en vía de desarrollo (Pinzón, Briceño, Gómez \& Latorre, 2003), lo que constituye un objeto de investigación que es abordado desde diferentes ángulos y perspectivas (Mitjáns, 2001).

A esta problemática, se han asociado diferentes factores como los procesos migratorios, la cultura, las diferencias socioeconómicas de los habitantes y el lugar de residencia sea este rural o urbano (Pinzón et al., 2003). Por lo anterior la Policía de Colombia cuenta con una especialidad dentro de la institución que es la de infancia y adolescencia, que junto con el sistema Nacional de Bienestar Familiar de acuerdo a las funciones y competencias asignadas por la ley, deben garantizar la protección integral de los NNA, llevando a cabo procesos enmarcados en tres líneas de acción, cómo los son la prevención: la vigilancia, el control y quienes contribuyen en la disminución de factores que vulneran los derechos de los menores trabajadores (Pedraza \& Ribero, 2006).

Así mismo, en el código de infancia y adolescencia colombiano se plantea que se deben garantizar los derechos de los infantes y adolescentes y su contexto familiar, asegurando que reciban la protección integral necesaria para el restablecimiento de estos derechos, verificando además que las entidades responsables de restablecer los derechos de los NNA cumplan de manera permanente con el mejoramiento de su calidad de vida y la de sus familias (Ley 1098, 2006).

\section{El fenómeno}

Desde los postulados del construccionismo social se plantea la formación del conocimiento desde el interior del sujeto. Así se logra comprender el fenómeno social a partir de la lectura de la situación de la población infanto-juvenil que se encuentra en situación de trabajo. Es decir, el sujeto construye el conocimiento de la realidad a través de los mecanismos cognitivos que dispone, de manera que la actuación sobre la realidad se logra a través del conocimiento, siendo experimentada en las situaciones y objetos que finalmente serán transformados (Araya, Alfaro \& Andonegui, 2007).

Aunado a lo anterior, se tiene que el constructivismo se concibe como el conocimiento que supone una perspectiva relativa de la realidad; porque es una construcción subjetiva y deja de ser una realidad absoluta, como identidad independiente o externa a cada sujeto, es por esto que según como lo menciona Gergen, toda verdad adquiere su validez en el marco de la comunidad que la construye y la legitima como tal (López-Silva, 2013).

Es por esto que, según los antecedentes del paradigma constructivista, estos se encuentran en los trabajos de Lev Vygotsky, quien ve al sujeto como producto de una interacción con su medio. Es decir, como resultado de un proceso histórico, social y cultural; teniendo en cuenta qué se aprende, cómo, dónde y cuándo, para poder comprender las relaciones interpersonales de cada sujeto y del grupo en un contexto social determinado (Payer, 2005).

Históricamente este paradigma denota una búsqueda epistemológica sobre cómo se conoce la realidad, cómo se aprende, el origen, el desarrollo del conocimiento y la cultura (Ferreiro, s.f.); por lo que el Constructivismo Social dicta que éste se forma no 
solo de las relaciones ambiente-yo, sino que es el resultado de la sumatoria con el entorno social. En este sentido, es por lo que una propuesta en la que lo concibe como algo provisional contiene diferentes construcciones formadas a través de las negociaciones dentro de una comunidad (Payer, 2005).

Aunado a lo anterior, se tiene que la transformación del conocimiento desde una postura constructivista busca la comprensión de la realidad a partir de cotidianidad por medio de las narrativas. Por tanto, el sujeto que conoce y el sujeto conocido se reconstruyen y generan de una manera recíproca.

Es por esto que, el fenómeno a abordar desde la perspectiva, se ajusta con la comprensión de tal situación, en donde, se visibiliza tanto la cotidianidad del sujeto que conoce, en este caso, los investigadores. Así como, la de los sujetos conocidos, a saber, niños, niñas y adolescentes en situación de trabajo, en tanto que se afectan las realidades de los sujetos en cuestión a partir de las verbalizaciones que emergen de dicha relación. Por lo anterior, el constructivismo social ha sido puesto en cuestión desde múltiples espacios, casi todos naturalistas, en el sentido que da a este término (Pérez, 2005).

Frente a la idea constructivista de que nada viene dado en el mundo de la experiencia social, la reacción ha consistido en la reafirmación del contenido universalista de la biología, por lo que hay universales psico-cognitivos de la naturaleza humana que dan forma a conductas y modelos culturales que a nivel historio consolida este paradigma en una búsqueda epistemológica sobre cómo se conoce la realidad, cómo se aprende, el origen, el desarrollo del conocimiento y la cultura (Ferreiro, s.f.).

La posición por parte de Bestard (2009), con respecto a las críticas naturalistas, se fundamenta en la existencia de un elemento genérico previo a todo desarrollo cultural que es presupuesto como dado y fortalecido por algunas dimensiones de la experiencia humana construidas a partir de lo sobreviniente. Por lo que se deben tener en cuenta aquellos cambios que cada ser debe dar, estando sumergido en su sociedad, cambios que dan paso a nuevas ideas, por lo que instituciones como Child Adolescent Psychiatry Mental Health investigan la incidencia de los desórdenes mentales de los jóvenes y los factores que inciden en sus problemáticas (Schmeck, Boonmann \& Fegert, 2016).

Por otro lado, es necesario mencionar la satisfacción que reciben los menores referente a la consecución de dinero a través de sus actividades, las cuales son remuneradas mediante la fuerza económica que suple las necesidades mediáticas y compiten fuertemente con las actividades escolares (Barth, Lloyd, Christ, Champman, \& Dickson, 2008). Las actividades escolares no refuerzan igual que la libertad y la consecución de dinero, ya que el hecho de que los infantes provengan de familias de escasos recursos, aumenta la probabilidad de conseguir medios económicos en la infancia que quienes no sufren esta condición (McLoyd, 1998).

\section{Trabajo infantil}

Desde la revolución industrial los niños eran empleados en las fábricas de producción, siendo una de las principales causas la falta de capacidad económica para el sostenimiento familia, lo que obligaba a muchos niños a trabajar con el fin de suplir esas necesidades (Valencia, 2014).

Actualmente, el Ministerio de Trabajo en Colombia considera que toda actividad económica realizada por un menor de edad con o sin remuneración debe tomarse como trabajo infantil; actividad que es una de las que mayor detrimento tiene a futuro con el menor (Acevedo, Quejada \& Yáñez, 2011). Así mismo, Basu \& Van (1998) indican que se debe tener claro que las investigaciones respecto al tema obligan al observador a tener en cuenta la clase de trabajo, así como también, el desarrollo del menor para no incurrir en falencias de tipo factuales respecto a la identificación del fenómeno a abordar. Por su parte Baland \& Robinson (2000) aducen que la labor realizada por los menores trabajadores es ineficiente, incluso para los mismos padres y cuidadores, pero que son desarrolladas por los infantes únicamente como factores altruistas de aprendizajes en el desarrollo de los hijos como seres que viven en un mundo capitalista.

Desde una mirada histórica, los trabajos en los que se empleaban a los menores eran por ejemplo en 
las fábricas de algodón, en las que algunos de ellos perdían sus extremidades o eran aplastados o decapitados por maquinas. Aquellos que tenían un poco más de edad estaban ubicados para trabajar en fábricas de cerillas, en las que muchos desarrollaron fosfonecrosis a causa de los vapores del fósforo y los otros trabajaron en fábricas de vidrio. En estas fábricas los niños se quemaban con cierta regularidad o quedaban ciegos y si esto no fuese suficiente varios niños que trabajaban en talleres de cerámica eran vulnerables al polvo de arcilla venenoso. Todos estos niños trabajaban alrededor de 14 horas diarias ganando el $10 \%$ o $20 \%$ de lo que un adulto ganaba y los niños que trabajaban y no eran empleados en este campo, los ubicaban en las casas como empleados domésticos o en la construcción trabajando entre 13 y 16 horas (Ministerio del trabajo, s.f.).

Esta explotación laboral a los menores, propició el surgimiento de leyes, como por ejemplo en España, la ley de regulación del trabajo infantil expedida el 24 de julio de 1873, que buscaba proteger a los niños en situación de trabajo. Es en este contexto en donde se producen los primeros acercamientos a las prohibiciones de este tipo de prácticas. Algunos apartados importantes señalaban que los niños y las niñas menores de diez años no serían admitidos al trabajo en ninguna fábrica, taller, fundición o mina o que no se excedieran las cinco horas de trabajo diarias a los niños menores de trece años, ni el de las niñas menores de catorce, prohibición de trabajo en las noches. Que para efectos de esta ley, la noche empezaba a contarse a partir de las ocho y treinta.

Según Pavez (2012) afirma que los actores, en este caso los menores, no siempre son visibles ante la sociedad, por lo que el fenómeno queda escondido sin que sea posible actuar. Esto debido a que se genera figurativamente una cortina que esconde la problemática, debido a que el menor no hace notar los derechos que le son vulnerados. Además, las metas que se trazan los infantes cuando estan desarrollando sus actividades económicas se ven afectadas por la presencia de jornadas de trabajo que desestimulan el pensamiento hacia la consecución de metas académicas (Hamre \& Pianta, 2001). Así como se generan problemas de desarrollo a los infantes por el hecho de no asistir a la escuela, ya que el entorno en el que se desarrollan las actividades del infante no son las adecuadas para ese desarrollo intelectual a la luz de que los comportamientos pueden verse afectados por el hecho de no encajar en espacio y tiempo (Noble, Nomran \& Farah, 2005).

Si bien en este punto histórico se permitió el trabajo en niños y que trataban de proteger a los más pequeños de las actividades laborales, esta situación no se aleja de la actualidad, el trabajo infantil es una problemática latente, un ejemplo de esto se puede ver en México, en donde tan solo el $65 \%$ de los niños y niñas no son remunerados, mientras que el $6 \%$ reciben un salario mínimo (Sandoval, 2007).

\section{Trabajo infantil en Colombia}

Los altos niveles de informalidad laboral que se perciben en el ambiente colombiano, denotan la baja capacidad institucional para el afrontamiento de esta problemática, demostrando así, un bajo crecimiento a nivel económico del país debido a que las organizaciones informales tienen poco acceso a recursos del sistema financiero. Esta característica repercute en la poca expansión y nula capacidad de inversión en tecnología, siendo esta informalidad considerada como los empleados particulares que laboren en establecimientos que ocupen hasta diez trabajadores al igual que trabajadores de índole familiar y domésticos donde tienen participación los menores de edad (Cardenas \& Mejia, 2007).

Los patronos aprovechan esta situación para generar ingresos a quienes no cuentan con el beneficio de un trabajo estable, por lo que la tasa de desempleo depende no solo de la oferta laboral y de las plazas disponibles para su desarrollo, sino también de cuan dispuestos estén las personas en realizar las tareas o que sean ocupados en ellas (Arango \& Posada, s.f.). Es por lo anterior, que el subempleo en Colombia cobra importancia en la dinámica del mercado laboral por lo que se relaciona la calidad del empleo y el uso de la mano de obra y que ha generado cambios importantes a través del tiempo. Pues si bien, a principios de la década de los 90 se estableció esta modalidad laboral en eslabones bajos, hacia mediados de la misma década se 
presenta un alto incremento, puntuando en la crisis del 99 hasta el año 2000, pero que nuevamente aumenta en el año 2008 (Puyana et al., s.f.).

De acuerdo a esto, es importante mencionar que las causas por las cuales existen más demanda que oferta respecto a la mano de obra laboral, se gesta desde varios ángulos, en los que tiene injerencia. Por ejemplo, la migración colombiana se basa en dos dimensiones según lo mencionan Cardenas y Mejia (2007), una que es el desplazamiento interno y la segunda la migración internacional, siendo este el resultado de problemas como la violencia, la falta de oportunidades, conflictos económicos, consecuencia del conflicto armado, que termina siendo crisis humanitaria para el Estado Colombiano, situaciones que tienen repercusiones sobre el trabajo infantil (Ibañez \& Querubin, 2004).

Así mismo, existen tres tipos de fenómenos migratorios donde hay participación de menores, el primero acerca de los menores que se encuentran dentro de una familia que migra, seguido de los menores que migran de manera autónoma y finalmente, los niños se quedan en su lugar de origen y su familia migra (Casteras, 2009).

\section{¿Quiénes son los niños y niñas trabajadores?}

Según el informe de la Organización Internacional del Trabajo (OIT) (citado en Pedraza, 2008), en donde se refiere a las tendencias mundiales del empleo mundial, se catalogan como jóvenes al grupo entre 15 y 24 años de edad; dicho documento, reporta tendencias negativas del empleo juvenil pasó de un 51.6\% a un 47.3\% para el año 2005 (Pedraza, 2008). Es por esto, que dicha organización considera un niño económicamente activo entre los de 5 y los 17 años, quien labora y es remunerado en el trabajo familiar en el área de producción y procesamiento de productos primarios y en la producción de bienes y servicios para la venta o el consumo propio, siendo estas las ofertas de empleo las más accesibles.

Quienes son responsables del diseño de políticas públicas, definen el empleo de niños como la participación de estos en actividades perjudiciales para su bienestar, por lo que existen actividades que son aún peores como por ejemplo los trabajadores sexuales o pornografía infantil, quienes generan un daño mucho mayor (Bernal \& Cardenas, 2010).

Todas estas actividades afectan el desarrollo integral del niño, un proceso de perfeccionamiento del hombre que requiere del goce de bienes materiales en cuanto a su realización en todas las dimensiones, siendo estas la dimensión física, mental y emocional, y aunque también afectan sus derechos fundamentales, tales como la vida, la integridad física, la salud y la seguridad social, la alimentación equilibrada, su nombre, nacionalidad, tener una familia y no ser separados de ellos, el amor y el cuidado, la educación y la cultura, la recreación y la libre expresión de su opinión, Valderrama et al. (2014), además de los cuidados en el interior de las familias hacia los niños, son de vital importancia para que el fenómeno sea reducido o por lo menos sea más controlable (Sarsour et al., 2011).

\section{Niños y niñas trabajadores en Colombia}

La población de menores empleados en Colombia ha aumentado en volumen pero se ha reducido en su participación, ya que a comienzos de los 90 se estimaba unas 19.9 millones de personas menores laboraba y para el 2003 de 22.4 millones, por lo que para el 2015 hubo 23.8 millones que representa el $44.7 \%$ de los colombianos. Implica esto que en la medida que aumente la esperanza de vida y las tasas de crecimiento natural de la población bajen la participación que continuará en declive (Universidad Nacional de Colombia, 2004).

Las tasas de ocupación con participación de menores en las ciudades son bajas y relativamente altas en el sector rural. Los menores son ubicados en labores de ayuda a sus familiares, por diferentes razones, una de ellas es debido a la pobreza del hogar. Así, en un $21 \%$, otras, en un $60 \%$ de los niños varones de siete a once años que trabaja a razón de que el trabajo honra su hombría y lo aleja de los vicios, pero que se tornan jornadas largas entre las 27 y las 30 horas semanales en los sectores rurales y 14 horas en el sector urbano. 
Esto redunda en la inasistencia a la escuela, que se debe en primer lugar a la falta de motivación y segundo a las razones económicas, donde se sitúa en un lugar preponderante a las cuestiones laborales por encima de las académicas (Salazar, s.f.), por lo que las cogniciones sociales están ligadas a las metas de los jóvenes, por lo que se afectan en el momento en que no se tiene en una medida lineal de acuerdo a las estructuras mentales que se construyen en el interior de los grupos (Reschke, Walle \& Dukes, 2017).

El sistema de información sobre trabajo infantil (SITI) del Departamento Administrativo de Estadística DANE (2006) (citado en Casteras, 2009), menciona que la población de niños trabajadores ha pasado de 1,5 millones en el año 2001 a 1,1 millones en el año 2005, por lo que hubo una reducción del 30,5\% en 4 años (Casteras, 2009).

Una vez conocidas las cifras del fenómeno en Colombia, es importante anotar lo que la Legislación define el trabajo infantil a través del Código de la infancia y la Adolescencia establecido por la Ley 1098 de 2006, el cual se fundamenta en convenios internacionales, como el Convenio 138 de la Organización Internacional del Trabajo OIT, que trata de la edad mínima de admisión al empleo. Esta ley sostiene los límites de la actividad laboral infantil y señala que en los menores de 15 años sólo se pueden trabajar hasta 14 horas semanales, única y exclusivamente en actividades de tipo artístico, cultural, recreativo o deportivo.

Por otro lado, la misma ley fija que los adolescentes de 15 a 17 años tienen derecho a trabajar, pero requieren la autorización del inspector de trabajo o en su defecto del ente territorial local, sin que excedan las seis horas diurnas diarias para un total de 30 horas semanales, siendo el trabajo nocturno prohibido y de especial connotación que los menores indígenas quienes son autorizados por las autoridades de su comunidad para realizar un trabajo (Vargas, 2006), sustentadas en sus costumbres; por lo que los menores de 17 a 18 años, pueden laborar siempre y cuando no trabajen más de ocho horas diarias y 40 horas semanales (Ley 1098, 2006).

Es importante hacer un símil comparativo con el Código de la Niñez y Adolescencia de la República de Ecuador, en su artículo 82 determina que la edad mínima para el trabajo tasa los quince años para todo tipo de trabajo; incluyendo el servicio doméstico, sin exceder las 6 horas diarias en 5 días a la semana, siempre y cuando no limite el efectivo ejercicio de su derecho a la educación (Bastidas Tamayo, 2014).

Por tanto, los niños al pasar de la inactividad a la actividad económica se encuentran con situaciones a la entrada en el mercado de trabajo, muchas veces por su inexperiencia y corta edad redunda en escenas de discriminación, se replican en altas tasas de desempleo que padecen generalmente en jóvenes de familias más pobres (Baquero, Guataqui, \& Sarmiento, 2000).

\section{Legislación colombiana y el fenómeno}

Las acciones que ha tenido el estado colombiano referente al fenómeno se sitúan principalmente en la Constitución Política de Colombia de 1991, más exactamente en el artículo 44, en el que se menciona la protección hacia los niños en este tema y así mismo se resalta la importancia de la niñez para el Estado y la prevalecía de los derechos de ellos sobre la de los adultos y se protegerán de las diversas formas de abandono, maltrato, abuso sexual, explotación laboral y trabajos riesgosos.

Por otro lado, la ley 1098 de 2006 de infancia y adolescencia menciona en el artículo 35 la edad mínima de los adolescentes que están autorizados para trabajar a los quince (15) años, exigida una autorización expedida por el Inspector de Trabajo o, en su defecto, por el Ente Territorial Local (Congreso de la República de Colombia, 2006).

Los procesos para que un menor de 18 años pueda trabajar formalmente en una organización recibiendo los beneficios como sueldos dignos, vacaciones, prima, seguridad social y horas de trabajo están regulados por la ley colombiana. Por supuesto, teniendo en cuenta que el menor goce de tiempo para desarrollar otras ocupaciones como el arte y la educación para trascender su desarrollo intelectual, social y afectivo (UNICEF, s.f.).

Basado en lo expuesto anteriormente, el Ministerio del Trabajo en el año 2012 realizó una campaña con 
el propósito de concientizar a la comunidad en la erradicación del trabajo infantil, de esta forma se construyó un audio que fue trasmitido en la emisora regional y se distribuyeron 1000 afiches con el tema ya antes mencionado.

Finalmente, el Ministerio del Trabajo, el Instituto Colombiano de Bienestar Familiar (ICBF), las Comisarías de Familia, entre otras, en su rol de velar por todos los niños, niñas y adolescentes a partir de la normal se involucra como un ente supervisor para garantizar como lo establece la norma la prohibición del trabajo infantil peligroso.

\section{Método}

\section{Tipo de investigación}

La presente investigación se sitúa bajo una metodología de tipo cualitativo, con un alcance descriptivo, a través de un proceso interpretativo de la documentación, la cual maneja un diseño de investigación y acción, por lo que Hernández, Fernández \& Baptista (2010) establece que la finalidad de la investigación acción es "propiciar el cambio social, transformar la realidad y que las personas tomen conciencia de su papel en ese proceso de transformación" (p. 509).

Es por esto, que la investigación buscó generar un cambio o una mejora en la calidad de vida de las personas de la sociedad, las cuales están incluidos en una situación de problemas que genera el medio en el que está inmerso. De este modo, se incluye un diseño transversal puesto que recopilan datos en un momento único como lo fue en la realización de las entrevistas semiestructuradas a las poblaciones definidas (Hernández et al., 2010).

Es así como se pretenden abarcar varios grupos y subgrupos de personas, en los cuales se obtiene información de diferentes situaciones en trabajo y un grupo de diferentes entidades las cuales realizaron para la visión del grupo focal.

Para el desarrollo del estudio, a través de la interpretación de las narrativas de los niños, niñas y adolescentes trabajadores, se manejó un diseño transicional descriptivo como lo plantea Hernández et al. (2010) pues se consideran útiles al momento de indagar a cerca de la incidencia de las modalidades, categorías o niveles de una o más variables en una población, siendo estudios descriptivos.

En todo el proceso de recopilación de datos y de información, se tuvo en cuenta el saber conceptual, el cual hace referencia a las habilidad para el manejo de conceptos, datos, informaciones y hechos, y después abre paso a el saber procedimental que se relaciona con la habilidad para ejecutar una acción según metas y técnicas adecuadas a la resolución de una tarea concreta y por último, se encuentra el saber actitudinal, el cual es concerniente a la habilidad para vincular el saber y el saber hacer a valores, principios o normas que configuran actitudes, todos estos saberes aseguran un bienestar para el desarrollo y el proceso de modificación y manejo de leyes y normas (Modelos pedagógicos, 2012).

\section{Población}

El proceso cualitativo es propositivo, por lo que la elección de la muestra fue llevada a cabo desde el planteamiento mismo del contexto en el que se realiza la investigación a través de los objetivos formulados (Hernández et al., 2010).

Es así, que para el análisis de la información se dividió la población en dos grupos focales: el primero constó de NNA trabajadores, de los cuales en primer lugar se identificaron los ubicados en los semáforos en la intersección de la carrera 40 con calle 15 entre los centros comerciales Primavera Urbana y Villacentro de la ciudad de Villavicencio, para un total de 8 participantes, cuyas edades oscilaban entre los 12 y los 16 años. Y segundo, aquellos niños, niñas y adolescentes que trabajaban en Pinares del Oriente con una población total 24 niños que se encontraban entre los 5 y 13 años.

El segundo grupo focal fue tomado a partir de las instituciones que conforman el Comité de Erradicación del Trabajo Infantil (CETI); el cual se encuentra conformado por 24 instituciones en las que se incluyen la Alcaldía de Villavicencio, la Secretaria de Gestión Social y Participación Ciudadana, la Secretaria de Gobierno, la Secretaria 
de Salud Municipal, la Secretaria de Educación, la Secretaria de Planeación Municipal, la Secretaria de Desarrollo y Competitividad Municipal, la Universidad Tecnológica de los Llanos (UNILLANOS), la Cámara de Comercio de Villavicencio, el Instituto de Turismo Municipal, la Corporación de Cultura Municipal de Villavicencio (CORCUMVI), el Instituto Municipal del Deporte y Recreación (IMDER), el Instituto Colombiano de Bienestar Familiar (ICBF) - Centro zonal 1 y 2, el Ministerio de Trabajo, el Servicio Nacional de Aprendizaje (SENA), Protección de Servicios Especiales (MEVIL), el Grupo de Infancia y Adolescencia, la Escuela de Carabineros Eduardo Cuevas García, Protección Social Eduardo Cuevas García, la Personería Municipal, la Caja de Compensación Familiar (COFREM), Prosperidad Social y la Secretaria de Comunicaciones.

\section{Instrumentos}

Para la recolección de la información se tuvo en cuenta como instrumento de apoyo un guion en el que se formularon los temas de interés para la investigación. Esto con el fin de recolectar la mayor información posible, dado que no se cuenta con información previa acerca de las posibles respuestas que se obtendrían; este instrumento de recolección sirvió ya que se obtuvo información acerca de opiniones de acuerdo a las cogniciones de los sujetos y los motivos de comportamiento (Hernández et al., 2010).

Con el fin de mitigar errores, se llevaron a cabo las entrevistas entre varios investigadores quienes, al momento de analizar las respuestas aportadas por los entrevistados, dieron su interpretación con el fin de que el sesgo fuera mitigado en aras de generar una mejor y más objetiva interpretación.

Las preguntas fueron elegidas por los investigadores previamente de acuerdo con el objetivo de la investigación.

\section{Resultados}

Como resultado de la aplicación de la herramienta utilizada para recolectar la información, se plantearon tres dimensiones para el grupo de NNA; fami- liar, trabajo y personal. Estas dimensiones a su vez estaban subdivididas en áreas como la edad, tipo de familia, convivencia ocupación de los familiares, remuneración gastos, escolaridad e intereses y para el grupo focal CETI, las dimensiones; trabajo, empleador y motivaciones; con las que se logra obtener la dinámica relacional de las narrativas entre uno y otro grupo focal.

\section{Grupo focal niños}

\section{Dimensión familiar}

Las familias monoparentales son las predominantes en la población analizada, lo que incide en que vivir con uno de los progenitores se convierte un factor de incidencia en la actividad laboral de los menores, puesto que la ausencia de uno de los proveedores económicos de la familia se debe suplir con uno o varios de los miembros constitutivos de ella.

Es así, que la predominancia en las manifestaciones de los actores respecto al tema familiar, muestra una fuerza discursiva hacia la figura materna como eje dinamizador del núcleo, y aunque existe figura paterna constituida por la presencia de la pareja de la madre (padrastro) hay una marcada jerarquía respecto a las responsabilidades a partir la presencia de varios hijos dentro de la estructura familiar. Por lo que los hijos deben proveer económicamente dependiendo de la posición de nacimiento. Así, por lo que entre mayor sea el individuo mayor será su aporte económico.

Aunado a esto, los hijos tienen responsabilidades propias de la figura ausente, que en la mayoría de los casos reportan la figura paterna. Es así que se encargan de la crianza de los hermanos menores y éstos de los quehaceres domésticos, en tanto, no cumplen con algunos requisitos para lograr aportes económicos, tales como, estatura, fuerza muscular, y en algunos casos la edad.

Esto representa una dinámica familiar en torno al lucro económico, dejando de un lado los estadios del desarrollo de los sujetos, por lo que la urgencia es la de suplir necesidades económicas y dejando a un lado las necesidades afectivas, sociales, culturales, académicas, etc. 
Por su parte los padres tienen diferentes tipos de ocupaciones como ventas ambulantes, oficios varios y construcción (civil), la mayoría reporta que las madres son ama de casa.

\section{Dimensión trabajo}

De acuerdo con la información aportada por los NNA, manifestaron que llevaban a cabo oficios como recolectar basuras en los alrededores de su lugar de residencia para luego ser llevada a las zonas donde el servicio público recolector de basuras transita, siendo esta la actividad que mayormente realizan.

Por otro lado, mencionan actividades como transporte de agua, actividad que realizan en apoyo a las familias vecinas desde el establecimiento comercial donde es adquirido el producto hasta la vivienda del consumidor. Reportan actividades de apoyo en la mano de obra en construcción (civil), siendo ésta la mejor remunerada según lo afirma la mayoría, aunque es a la que menor acceso tienen por lo que es un ideal el llegar a conseguir ocuparse en esta. En esta dimensión se ubicó el factor de la remuneración económica producto de los trabajos llevados a cabo por los NNA, la cual oscila entre los $100 \mathrm{y}$ los 25.000 pesos dependiendo de la actividad, por lo que manifiestan que reciben el dinero una vez finalizan la acción.

Los participantes mencionan que el dinero ganado es entregado a sus cuidadores y el restante lo usan para ocio como video juegos y comprar golosinas.

\section{Dimensión personal}

En este apartado se ubican características de los NNA a partir de su etapa del desarrollo entre los 6 y los 13 años, mencionan cursar entre segundo de primaria y primero de bachillerato. Así mismo, aducen utilizar como actividades de ocio, jugar video juegos, practicar futbol y ver televisión.

\section{Grupo focal Comisión Erradicación del Trabajo Infantil (CETI)}

\section{Dimensión trabajo}

Teniendo en cuenta la metodología utilizada y las herramientas de recolección de la información, se logran determinar los tipos de trabajo ejercidos por los NNA en la ciudad de Villavicencio a partir de los criterios de valoración expuestos por los integrantes de las instituciones del CETI.

Es por lo que los funcionarios refieren como actividades de trabajo infantil dentro de la ciudad de Villavicencio aquellas como las ventas ambulantes, generalmente en los semáforos y la mendicidad.

\section{Dimensión empleador}

Respecto a las personas que pagan por las actividades que realizan los NNA, los funcionarios del comité refieren a estas personas como ambiciosas, explotadores, empresas informales y personas ocultas reportadas como delincuentes.

\section{Dimensión motivaciones}

Finalmente, en esta dimensión los participantes dan a conocer sus impresiones respecto a lo que en su devenir profesional conocen respecto a que motiva que los NNA trabajen y generen ganancias económicas, por lo que dan cuenta de que los factores se basan en la falta de recursos económicos y colaboración hacia las familias, aspectos culturales, motivaciones económicas, gusto y por presión familiar.

\section{Comentarios}

Es importante tener en cuenta, que al realizar una comparación sistemática de la literatura y las realidades encontradas en la investigación se enlazan una con la otra, por lo que la teoría muestra las diferentes formas de trabajo infantil. Esto responde a la pregunta de investigación basada en lo que Cutri et al. (2012) mencionan respecto a que el fenómeno fue comprendido desde la lectura de las 
vivencias de los intervinientes, quienes afirman conocer una realidad enmarcada en unas actividades que para ellos y para sus cuidadores hace parte de su cultura y que estas forman relaciones para el entorno y las personas como provistas de manera mediática sobre sus interacciones dentro de su comunidad (Jurado, Olmos \& Pérez, 2014). En consecuencia, esto dista del conocimiento que tienen las instituciones del Estado colombiano respecto a esta realidad, por lo que es importante revisar los programas de asistencia en la erradicación del fenómeno y estos ser contrastados con lo que sucede en la realidad.

A través de las verbalizaciones de los NNA se pudo observar que las actividades lucrativas eran propias de sus vivencias, por lo que son observadas de manera natural al momento de disponer de los recursos para comenzar a proveer el hogar económicamente, tal como se muestra en las dimensiones familiares y trabajo, visto desde la revolución industrial. El fenómeno está presente y éste no se ha desligado hoy en día, pues son las mismas necesidades que se siguen reportando tales como la falta de sustento económico para el sostenimiento familiar (Montoya, 2006), tal como se pudo ver en la dimensión motivaciones por parte de los funcionarios del CETI, en la que son conocedores de la problemática y que también está presente en la dimensión familia de los NNA.

Es así que, basado en que el fenómeno fue observado desde una perspectiva constructivista, donde se logra identificar la comprensión de los actores a partir de las verbalizaciones por medio de la observación en dos grupos focales, sin que ellos sean conocedores de que sus derechos están siendo vulnerados, los cuidadores de los NNA mantienen dentro de sus dinámicas familiares una provisión económica, fracturan los procesos de desarrollo de los niños involucrándolos en actividades que no son propias de sus etapas.

Por otro lado, es importante resaltar que la información tanto teórica como de las manifestaciones de los funcionarios del CETI son concordantes en que para llevar a cabo los diferentes oficios en los que son empleados los menores hoy en día no han cambiado a través de la historia. Por el contrario, se basan en la participación en empresas de índole familiar, empleos domésticos, por lo que los patronos aprovechan la situación de la inestabilidad laboral y la falta de mecanismos de consecución de dinero para que los menores sean empleados en actividades de índole comercial siendo remunerado el menor según lo menciona la OIT (Arango \& Posada, s.f.). Esto es algo que no es acorde a la realidad observada, pues los integrantes del CETI ven a los empleadores como una estructura delincuencial que no tiene que ver con los lazos familiares, dedicada a la explotación de los NNA con el fin de generar ingresos económicos y por otro lado los NNA afirman que quienes proveen el lucro económico son personas ajenas a su familia, quienes se benefician de las actividades que llevan a cabo los menores y son ocasionales. Lo que quiere decir, es que no hay un contrato laboral especifico sino que se trata de una simple remuneración económica por un favor realizado.

Se resalta lo mencionado por Valderrama et al., (2014), quienes afirman que al realizar este tipo de actividades, el menor se ve afectado en su desarrollo integral, por lo que se logra observar la conexión con la realidad, donde los NNA entrevistados prefieren trabajar que estudiar y le dan un peso muy fuerte a la provisión económica más que a su capacitación. Esto redundará a futuro en un adulto que no tiene una formación sólida, sin mencionar que no se cuenta con programas contundentes para el paso de ser menor de edad a la adultez en términos de situación laboral por parte del Estado (Salazar. s.f.).

Aunado a lo anterior, no solamente es necesario que las condiciones en las que nace un niño sean aptas para su desarrollo; las cuales tienen influencia en los comportamientos durante las diferentes etapas de la vida, sino también que desde sus estructuras iniciales de aprendizaje en la escuela los menores formen una conjugación cognitiva de lo que significa el trabajo y cómo es la forma de desarrollarlo. Este aspecto redunda en que la problemática es de vital importancia para que sea tomada desde una perspectiva real y que se fortalezcan los programas de intervención y erradicación del fenómeno (Pianta \& Stuhlman, 2004). 
Las mismas familias son las que incentivan el trabajo infantil en las sociedades y que en muchos casos, son estas las circunstancias por las cuales los menores toman la decisión de salir de sus casas, ya que cuentan con la competencia para conseguir dinero y les es más fácil salir del seno de su hogar que continuar con el seguimiento de reglas que le acarrean dificultades de disciplina y gobierno propio. Este resultado genera daños físicos y emocionales al menor al estar expuesto a las vivencias de la calle, es por lo que se observa en la dimensión familia, en la que los niños reportan la necesidad de proveer su núcleo a partir de actividades lucrativas.

Con una connotación especial, la legislación colombiana posee una estructura administrativa en la materia, lo cual es de conocimiento a través del Código de Infancia y Adolescencia (Ley 1098 de 2006), pero que no hay un efecto contundente en dicha labor, pues las significaciones aportadas por parte de las instituciones en algunos casos distan de la realidad que se presenta (Mogica, 2011).

Es importante mencionar que, las instituciones encargadas de la erradicación del trabajo infantil conozcan de la realidad que se presenta con respecto al fenómeno y sus manifestaciones, ya que las actividades que realizan pueden tener buenos medios, pero su fin no es de efectividad total para bienestar de la población estudiada.

Finalmente, la cultura en la cual se desarrolló el estudio muestra que no solamente las instituciones encargadas de la erradicación del trabajo infantil tienen responsabilidad en el fenómeno, sino que también otras donde se gesta la problemática, ya que la lógica del común de las personas, según lo reportado, es que es normal es que los menores trabajen y aporten provisiones económicas a sus hogares, lo cual se puede mitigar desde una mirada pedagógica a partir de las diferentes entidades que tienen participación en ello (Ministerio de Trabajo República de Colombia, 2012).

\section{Referencias}

Acevedo, K., Quejada, R., \& Yánez, M. (2011). Determinantes y consecuencias de trabajo infantil. Un análisis de la literatura. Revista Facultad de Ciencias Económicas, 19(1), 113-124. Doi: https://doi.org/10.18359/rfce.2263

Arango, L. E. \& Posada, C. E. (s.f.). La participación laboral en Colombia. Revista Coyuntura Social, 63-83.

Araya, V., Alfaro, M., \& Andonegui, M. (2007). Constructivismo: orígenes y perspectivas. Laurus, (13) 76-92.

Asamblea Nacional Constituyente. (1991). Constitución Política de Colombia. Título 2 De los Derechos, las Garantías y los Deberes Capitulo 2. De los derechos sociales, económicos y sociales. Art 44. Recuperado de http:// www.constitucioncolombia.com/titulo-2/ capitulo-2/articulo-44

Avella, A. C. \& Medina., R. R. (2006). El trabajo infantil y juvenil en Colombia y algunas de sus consecuencias. Revista Latinoamericana de Ciencias Sociales, Niñez y Juventud. Recuperado de http://bibliotecadigital.udea.edu.co/bitstream/10495/6493/1/VivianaCano_2013_didacticatic.pdf

Baland, J. \& Robinson, J. (2000). Is Child Labor Inefficient?. Journal of Political Economy, 108 (4), 663-679. Doi: https://doi.org/10.1086/316097

Barth, R., Lloyd, E., Christ, L., Champman, M., \& Dickson, S. (2008). Child Walfare Workers Characteritics and Job Satisfaction: A National Study. National Association of social Workers, 199-209 recuperado de http://s3.amazonaws. com/academia.edu.documents/40106381/ Barth_et_al_2008_Child_welfare_worker_characteristics_and_job_satisfaction_-_A_national_study.pdf?AWSAccessKeyld=AKIAIWO 
WYYGZ2Y53UL3A\&Expires $=1491593667 \&$ Sig nature=Nu1S3SHD\%2BsMnrk3FQaGaewtRqB I\%3D\&response-content-disposition=inline\% 3B\%20filename\%3DChild_Welfare_Worker_ Characteristics_and.pdf

Bastidas Tamayo, F.V. (2014). Responsabilidad penal del progenitor tutor o curador de un menor frente a la circunstancia de obligarlo a trabajar o a mendigar (Trabajo de grado). Universidad Central de Ecuador. Recuperado de http://www. dspace.uce.edu.ec/bitstream/25000/3220/1/TUCE-0013-Ab-142.pdf

Basu, K. \& Van, P. (1998). The Economics of Child Labor. The American Economic Review, 88(3), 412-427.

Bernal, R. \& Cardenas, M. (2010). Trabajo infantil en Colombia. FEDESARROLLO, USAID-Colombia Productiva. Recuperado de http://www. repository.fedesarrollo.org.co/bitstream/ handle/11445/151/CDF_No_48_Marzo_2014. pdf?sequence $=3$ \&isAllowed $=y$

Bestard, J. (2009). Revista de Antropología Social entre el esencialismo biológico y el constructivismo social. Revista de Antropología Social, 18, 83-95.

Cardenas, M. \& Mejia, C. (2007). Informalidad en Colombia: Nueva Evidencia. Documento de trabajo, Banco Interamericano de Desarrollo. Recuperado de http://www.repository. fedesarrollo.org.co/bitstream/handle/11445/ $805 /$ ?sequence $=1$

Casteras, D. (2009). Efectos de la migración sobre el trabajo infantil en Colombia. Revista económica institucional, 11(20), 229-252.

Congreso de la República. (8 de noviembre de 2006) Código de la Infancia y la adolescencia. [Ley 1098 de 2010].

Congreso de la República. (5 de agosto de 1950) Código sustantivo del trabajo. [Ley 2663 de 1950]. Ed. Legis.
Cutri, A., Hammermuller, E., Zubieta, A., Muller, B., \& Miguelez, L. (2012). Trabajo infantil: una problemática social que nos compromete. Archivos Argentinos de Pediatría, (4), 350-358. Doi: https://doi.org/10.5546/aap.2012.350

Hamre, B. \& Pianta, R. (2001). Early Teacher-Child relationships and the Trajectory of Children's School Outcomes through Eighth Grade. Child Development, 72(2), 625-638. Doi: https://doi. org/10.1111/1467-8624.00301

Hernández, R., Fernández, C., \& Baptista, P. (2010) Metodología de la Investigación. $3^{a}$ edición. México: Mc Graw Hill.

Ibañez, A. M. \& Querubin, P. (2004). Acceso a tierras y desplazamiento forzado en Colombia. Documento Cede, 23, 1-114.

Jurado, P., Olmos, P., \& Pérez, A. (2014). Los jóvenes en situación de vulnerabilidad y los programas formativos de transición al mundo del trabajo. Educar, 51(1), 211-224.

López-Silva, P. (2013). Realidades, Construcciones y Dilemas. Una revisión filosófica al construccionismo social. Cinta Moebio, 46, 9-25.

Magnobosco, M. (2014). El construccionismo social como abordaje teórico para la comprensión del abuso sexual. Revista de psicología, 32(2), 1-23.

McLoyd, V. (1998). Socioeconomic Disadvantage and Chil Develpment. The American Psychologist, 53(2),185-204. Doi: https://doi. org/10.1037/0003-066X.53.2.185

Ministerio de Trabajo. (s.f.). Despertando conciencia junto a la sociedad civil para la prevención del trabajo infantil. UNICEF. https://www. unicef.org/argentina/spanish/despertando_ conciencia.pdf

Ministerio de Trabajo República de Colombia. (2012). Desarrollo de la política para la prevención y erradicación del trabajo infantil. Colombia. 
Modelos pedagógicos. (2012). Obtenido de modelos constructivistas: http://modelospedagogicos. webnode.com.co/modelo-constructivista/

Mogica, J. (Diciembre de 2011). Universidad Autonoma del Estado de Hidalgo. Recuperado de http://www.uaeh.edu.mx/docencia/P_ Presentaciones/huejutla/administracion/temas/tipos_de_empleo.pdf

Montoya, J. (2006). Menores en situación de calle en la ciudad de Toluca. Papeles de población, 12(48), 247-280.

Mitjáns M. A. (2001). Trabajo Infantil y Subjetividad: una perspectiva necesaria. Estudios de Psicología, 6(2), 235-244. Doi: https://doi. org/10.1590/S1413-294X2001000200011

Noble, K., Nomran, M., \& Farah, M. (2005). Neurocognitive correlates of socioeconomic status in kindergarten children. Developmental Science, 8(1), 74-87. Doi: https://doi. org/10.1111/j.1467-7687.2005.00394.x

Nova, P. (2008). Trabajo infantil; los riesgos laborales en situaciones legalmente prohibidas y sus consecuencias para la salud y la seguridad. Revista Médica de Seguridad en el trabajo, 54(213), 9-21.

Payer, M. (2005). Teoría del constructivismo social de Lev Vygotsky en comparación con la teoría Jean Piaget. Universidad Central de Venezuela Facultad de humanidades y educación escuela de educación departamento de psicología educativa Cátedra de Psicología Educativa. Recuperado de http://www.proglocode.unam.mx/system/files/TEORIA\%20DEL\%20 CONSTRUCTIVISMO\%20SOCIAL\%20DE\%20LEV\%20 VYGOTSKY\%20EN\%20COMPARACI\%C3\%93N\%20 CON\%20LA\%20TEORIA\%20JEAN\%20PIAGET.pdf

Pavez, I. (2012). Sociología de la infancia: las niñas y los niños como actores sociales. Revista de Sociología, (27), 81-102.

Pedraza, A. C. (2008). El mercado laboral de los jóvenes y las jóvenes de Colombia: realidades y respuestas políticas actuales. Revista
Latinoamericana de Ciencias Sociales Niñez y Juventud, 6(2), 853-884.

Pedraza, A. C. \& Ribero., R. (2006). El trabajo infantil y juvenil en Colombia y algunas de sus consecuencias claves. Revista Latinoamericana de Ciencias Sociales, Niñez y Juventud, 4(1), 1-31.

Pérez, R. C. (2005). Elementos básicos para un constructivismo social. Avances en Psicología Latinoamericana, 23(1), 43-61.

Pianta, R. \& Stuhlman, M. (2004). Teacher-Child Relationships and Children's Success in tge First Years of School. School Psychology Review, 33(3), 444-458.

Pinzón A. M., Briceño L., Gómez A. I., \& Latorre C. (2003). Trabajo infantil en las calles de Bogotá. Revista Ciencias de la Salud, 1(2), 151-63.

Puyana, R., Ramos, M. A., \& Zarate, H. (s.f.). Deterinantes del subempleo en Colombia: Un enfoque a través de la compensación salarial. Banco de la República. Banco de la República. Romero.

Reschke, P., Walle, E., \& Dukes, D. (2017). Interpersonal Development in Infancy: The Interconnectedness of Emotion Understanding and Social Cognition. Child Development Perspectives, 11(3), 178-183. Doi: https://doi. org/10.1111/cdep.12230

Sandoval, A. (2007). Trabajo infantil e inasistencia escolar. Revista Brasileira de Educacao, 12(34), 68-80. Doi: https://doi.org/10.1590/S141324782007000100006

Salazar, M. C. (s.f.). El trabajo infantil en Colombia: Tendencias y nuevas políticas. Defensa de los Derechos de los niños, Bogotá. Recuperado de http://biblioteca.clacso.edu.ar/Colombia/ alianza-cinde-umz / 20160629073430 / ErnestoDuranStrauch.pdf

Sarsour, K., Sheridan, M., Jutte, D., Nuru-Jeter, A., Hinshaw, S., \& Boyce, T. (2011). Family Socioeconomic Status and Child Executive Functions: The Roles of Language, Home 
Environment, and Single Parenthood. Journal of the International Neuropsychological Society, 17, 120-132. Doi: https://doi. org/10.1017/S1355617710001335

Schmeck, K, Boonmann, C., \& Fegert, J. (2016). Thematic series CAPMH "Forensic Child and Adolescente Psychiatry and Mental Health". Child and Adolescent Psychiatric Research Deparmet, Psychiatric University Hospital Basel. Switzerland. DOI 101186/s 13034-016-0126-3.

UNICEF. (s.f.). unicef. Obtenido de http://www.eird. org/cd/toolkit08/material/otros/derechos_de_ los_ninos/articulos.pdf

Universidad Nacional de Colombia. (18 de 11 de 2004). Situación actual y prospectiva de la niñez y la juventud en Colombia. Pp. 1-103.
Valderrama, Y.A., Salgado, D.A., \& Ortiz, J. (2014). Identificación de los factores que conllevan a niños, niñas y adolescentes en edades comprendidas entre los 9 a 17 años a trabajar en las plazas de mercado de la ciudad de Florencia (Trabajo de grado, Universidad Nacional Abierta y a Distancia. Recuperado de http://repository.unad.edu. co/bitstream/10596/2492/1/52159470.pdf

Valencia, J. (2014). Ser niño en situación de calle: un riesgo permanente. Revista Facultad Nacional de Salud Pública, 32(2), 85-91.

Vargas, S. (2006). El papel de los niños trabajadores en el contexto familia. El caso de migrantes indígenas asentados en el Valle de San Quintín. Revista papeles de población, 12(48), 227-245. 